\title{
XXVIII. The origin of the power which causes the stellar radiations
}

\section{Jacob Ennis}

To cite this article: Jacob Ennis (1878) XXVIII. The origin of the power which causes the stellar radiations, Philosophical Magazine Series 5, 6:36, 216-225, DOI: 10.1080/14786447808639499

To link to this article: http://dx.doi.org/10.1080/14786447808639499

曲 Published online: 13 May 2009.

Submit your article to this journal $\pi$

Џll Article views: 2

Q View related articles $\sqsubset$ 
mercury, which is stopped by the stratum of mercury-salt which is formed, and which enables the lead particles to reassert their electropositiveness and be further operated upon. Then a fresh diffusion ensues and the play is repeated.

\section{The Origin of the Pover which causes the Stellar} Radiations. By JАCOB ENNIs*.

TT is important for scientific men to acquire the habit of 1 regarding all matter as having been diffused equally, or nearly equally, through all space. This is the initial point in our history of creation, the earliest period which we know. From this universal diffusion we can trace, by the operation of well-known forces, the origin of the stars. We can understand how the vast sidereal systems, stellar nebulæ, were formed, and how they must remain stationary in space. We can understand the origin of solar and planetary systems, and how these latter systems are all moving through space with inconceivable velocities. We can learn (as I proved in my paper in the 'Philosophical Magazine' for April 1877) that gravity is the force which imparted their velocities to all the stars and to all stellar systems. And now I am to prove that in this same universal diffusion of all matter wo can behold the reservoir, the illimitable reservoir, of that force which radiates the undulations of heat, light, and actinism from all the stars. I will point out also that, in the condensation from that primitive diffusion, we can behold the origin of those modifications of matter which we call the simple elements. In that condensation also we can see the first manifestations of several of the physical forces, such as electricity, magnetism, cohesion, heat, and light. The three primitive forces, of whose origin we know nothing, are repulsion, chemical force, and gravity.

The diffusion of matter in a gaseous form, by the ordinary repulsive force, is a store of heat; and this store is greater or smaller in proportion to the amount of diffusion. It is analogous to the removal of a pound weight from the surface of the earth : the further it is removed the greater will be the amount of heat produced by its fall. It has been found that when our atmospheric gases expand from one volume to two, their temperature is lowered, and just $144^{\circ} \mathrm{F}$. of heat are absorbed and rendered latent. Every addition of the original volume by expansion renders latent an additional $144^{\circ}$ of heat; that is, so much heat loses its character and form of heat, and it reappears as repulsion or diffusion. When compressed again, that

* Communicated by the Author. 
diffusion is annihilated, and the same original amount of heat is reproduced. By a thermometer in the receiver of an airpump the loss of heat by expansion is seen clearly enough; but in the sinking of an oil-well recently in North-western Pennsylvania it was manifest on a magnificent scale. Instead of striking a reservoir of oil, the auger entered an accumulation of gas (a hydrocarbon); and as this gas expanded on issuing with amazing force from the orifice of the well, its own heat was converted into repulsion, and it absorbed the heat from the surrounding atmosphere and from the ground. The watery vapour in the gas and in the air fell down as snow, and the ground all around was frozen : it was like the freezing of carbonic acid by its own expansion.

From the well-ascertained fact that a gaseous diffusion absorbs $144^{\circ} \mathrm{F}$. of heat by every increase of its original volume, which heat is converted into the form of repulsion, and is called latent heat, we are enabled to calculate the amount of latent heat in all the higher strata of our atmosphere. A volume of air rising three and a half miles (more accurately 3.43 miles) becomes two volumes, and contains $144^{\circ}$ of latent heat; on rising double that distance ( 6.86 miles) it becomes 4 volumes; therefore 3 new volumes are added, and it contains $432^{\circ}$ of latent heat; at 10.29 miles it becomes 8 volumes, and 7 new volumes are added, and therefore it contains $1008^{\circ}$ of latent heat; and so on upward, according to the following Table ( $p$. 218), which (with other columns here omitted) was constructed by Mr. Benjamin V. Marsh, merchantand, like Benjamin Franklin, an amateur of science in Philadelphia. It first appeared in the 'American Journal of Science,' July 1853. His paper on many accounts is valuable; and the subject is further carried out in my own paper on Meteors in the Proceedings of the American Association for the Advancement of Science for 1871.

The appearance of meteors is a proof that our atmosphere extends upwards more than 200 miles, and that its loftier regions are wonderfully charged with latent heat. In my paper on Meteors I gave two instances, one in Europe and the other in America, where meteors travelled more than a thousand miles through the air from 40 to 100 miles high, and continued vividly bright through the whole distance until they passed from view. Last year another passed in the same incandescent manner a thousand continuous miles over the United States. I also gave other instances of vertical descents, when invariably the light of the meteors went out before reaching the ground. They prove that in these cases the very bright light comes, not from the meteor itself, but from the air in its 
front, which is compressed and made to give out its abundant latent heat. Had the meteors themselves been so vividly incandescent, their brightness would have continued another twinkling of an eye before they struck the earth. Had friction been the cause of the heat, their light would have become brighter in the dense lower atmosphere, where invariably their light goes out, albeit gravity hastens their velocities. I pointed out in that paper the fallacies in the reasonings of high authorities who thought they had proved that meteoric light is due to friction.

\begin{tabular}{|c|c|c|c|}
\hline $\begin{array}{l}\text { Height, } \\
\text { in miles. }\end{array}$ & $\begin{array}{l}\text { Number of volumes } \\
\text { corresponding to } \\
\text { 1 volume at the surface } \\
\text { of the earth. }\end{array}$ & $\begin{array}{l}\text { Number of degrees of } \\
\text { latent heat. }\end{array}$ & $\begin{array}{l}\text { Number of grains } \\
\text { of air in cylinder } \\
\text { 1 mile long and } \\
\text { 1 foot in diame- } \\
\text { ter. Weight at. } \\
\text { surface of the } \\
\text { earth }=2942817 \\
\text { grains, }=334 \cdot 69 \\
\text { pounds avoirdu- } \\
\text { pois. }\end{array}$ \\
\hline $3 \cdot 43$ & 2 & 144 & 1171424 \\
\hline $6 \cdot 86$ & 4 & 432 & 585712 \\
\hline $10 \cdot 29$ & 8 & 1008 & 292856 \\
\hline 13.72 & 16 & 2160 & 146428 \\
\hline $17 \cdot 15$ & 32 & 4464 & 73214 \\
\hline $20 \cdot 58$ & 64 & 9072 & 36607 \\
\hline $24 \cdot 01$ & 128 & 18288 & 18303 \\
\hline $27 \cdot 44$ & 256 & 36720 & 9152 \\
\hline 3087 & 512 & $73 \overline{8} 84$ & 4576 \\
\hline $34 \cdot 30$ & 1024 & $1473 \perp 2$ & 2288 \\
\hline 37.73 & 2048 & 294768 & 1144 \\
\hline $41 \cdot 16$ & 4096 & 589680 & 572 \\
\hline 44.59 & 8192 & 1179504 & 286 \\
\hline $48 \cdot 02$ & 16384 & 2359152 & 143 \\
\hline $51 \cdot 45$ & 32768 & 4718448 & 72 \\
\hline $54 \cdot 88$ & 65536 & 9137040 & 36 \\
\hline $58 \cdot 31$ & 131072 & 18874224 & 18 \\
\hline $61 \cdot 74$ & 262144 & 37748592 & 9 \\
\hline $65 \cdot 17$ & 524288 & 75497328 & 4 \\
\hline $68 \cdot 60$ & 1048576 & 150994800 & 2 \\
\hline 102.90 & 1073741824 & 154618822512 & \\
\hline $137 \cdot 20$ & 1099511627776 & 158329674399600 & $\frac{1}{82 x^{2} 8 \pi}$ \\
\hline $171 \cdot 50$ & 1125899906842624 & 162129586585337712 & उ85- $\frac{1}{70912}$ \\
\hline 205.80 & 1152921504606846976 & 166020696663385964400 & $\begin{array}{c}586830912 \\
549755813888\end{array}$ \\
\hline
\end{tabular}

The point of most intense interest in the Table of Marsh is the wonderfully large amount of latent heat in an almost infinitesimally small amount of the air. Look at the lower line in the Table; how immense is the physical force! and how minute is the amount of matter! and yet the two are physically coupled. Here is a wouder which leads to the very gravest consequences. It is an image of the sun. The amount of matter in that great orb is infinitesimally small when com- 
pared with his physical force-that force which has sont out the solar radiations many millions of millions of years. Now mark the origin of that solar force. When our sun in its nebulous period was expanded less than halfway to the nearest fixed star ( $\alpha$ Centauri), it was $666,000,000,000,000,000$ times more rare than hydrogen. It was more rare than the highest strata of our atmosphere; and its repulsive force, latent heat, was in greater proportion than in the last line of the Table of Marsh. That force is indestructible. It cannot be lost. During the condensation of the sun it must be stored up in the solar elements, and pass off as solar radiation. I speak not now of the falling together of the materials of the sun by the force of gravity, and the consequent liberation of heat from that source. That is exceedingly small, not worth counting, although it might continue the solar radiations $20,000,000$ years.

But in the repulsive force which so vastly expanded the nobulous sun we behold only one of the sources of its present power. There was still another and a greater power then residing in our nebulous sun. It was a power great enough to overcome the repulsive force, to condense the nebulous sun to his present liquid condition, and to store up all that infinity of latent heat in the chemical elements of the sun. This paramount and overcoming power was the chemical force. When all matter was diffused through all space, its condensation could not have been caused from the loss of its heat by radiation-in common phrase, by "the cooling of the primitive fire mist." If all space had been thus filled by sensible heat (" fire mist)," then that heat must have remained. It could not radiate away; for there was no other space where it could go. Therefore we must look to some other well-known cause for the condensation of the primitive solar gases. The only other cause we can think of is chemical action; and that is an adequate, a normal, and a familiar cause. The chemical force, indeed, is the great condensing-power in the universe. Oxygen and hydrogen, when under its influence, are rapidly condensed nearly 2000 times in volume to form water: but the repulsive force of these gases is not lost; it is converted partly into heat and light, and partly into cohesion.

We will now attend to some well-known faets to illustrate how the chemical force may overcome the repulsive force, and imprison in a dense solid or a liquid vast stores of many kinds of physical forces. Gunpowder, nitroglycerine, dynamite, and mercurial fulminating powders are examples. In all these cases the original repulsive force has been overcome and appropriated by the chemical force. As being most familiar, we will confine our analysis to gunpowder. Its power is stored 
up in its chemical force, and in the repulsion of its three gases, oxygen, nitrogen, and carbonic acid. These gases were floating about in the air until the chemical force laid hold on them, and pressed them down into the small solid materials of the powder. The nitrogen and oxygen were quietly combined as nitric acid, and then united with the potassa of the earth, forming nitrate of potassa, or saltpetre. The gaseous carbonic acid was absorbed through the stomata of plants, then dissolved, and its carbon hardened into wood, or rather the charcoal of the wood. This saltpetre and charcoal, with a very little sulphur, are the only components of gunpowder. We know how the least spark liberates these imprisoned gases; and how terribly their native repulsive power shows itself, not only with thundering sound, but with the power of thunder, and with the heat of lightning.

The materials of gunpowder show this power, not only in the thunder-like explosion and the lightning-like heat, but also in vast stores of chemical force. One of the elements of saltpetre, composed of oxygen and nitrogen, is the violent aqua fortis, or nitric acid. Two other gases, hydrogen and chlorine, are combined and condensed by chemical force into a very different material called hydrochloric acid. These two acids when combined show their power by dissolving gold as a lump of sugar is dissolved in a cup of tea. Hydrogen and nitrogen also are combined and condensed by the chemical force into the violent ammoniacal gas. This ammonia combines with the nitric acid, and the two are hardened by the chemical force into a solid, the nitrate of ammonia. All these are striking instances of the way the repulsive force may be overcome and converted into the chemical force, this chemical force being lodged in vast abundance in small amounts of the resulting solids and liquids. How inert and comparatively feeble are the chemical properties of the four gases which form aqua regia (oxygen, hydrogen, nitrogen, and chlorine)! But the force residing in them in the form of repulsion is great beyond expression, beyond measurement. Think of the wonderful mechanical force lately found necessary to reduce them to a liquid condition-still retaining their repulsion and ever ready to burst out into their native gaseous state. As they can be so easily reduced to the solid condition by the chemical force, this is an impressive illustration of the great power of the chemical force in overcoming and appropriating to itself the repulsive force. The great idea here conveyed is that the repulsive force of these gases cannot be annihilated. It completely disappears as repulsion when these gases are solidified or liquified; but it is converted into the chemical force. 
Two great ideas are now clearly proved. First, the inconceivably large amount of repulsive force coupled with every single infinitesimally small portion of the sun when in the nebulous condition, as illustrated by the last line of the Table of Marsh. Secondly, the still greater amount of chemical force then residing in the nebulous sun, which overcame the repulsive force and compressed the sun to his present size-as illustrated by that same force when it compresses oxygen and nitrogen into nitric acid, and when it compresses oxygen and hydrogen into water-in the case of nitric acid, retaining a vast store of chemical force, and, in the case of water, producing a large amount of heat and also of cohesion, as I will soon show.

Both these stores of original force, the repulsive and the chemical, must now reside in the compressed sun, the same as great stores of force reside in compressed gunpowder. And no finite mind can pretend to say how long that reservoir of solar force may be able to send out the radiations from the sun. Count up millions of years as we may, we cannot begin to touch the problem. We stand before its immensity bowed in reverence, as when we contemplate the infinity of space and the eternity of time.

In this condensation of the nebulous stars, our sun included, we behold the origin of the so-called simple chemical elements. These are not eternal entities. They are mere modifications of the primitive gascous diffusion, formed successively as condensation went on. The proofs I cannot epitomize here, as they are already condensedly stated in my volume, "The Origin of the Stars,' where they occupy nearly the entire Second Part, more than forty pages. In the meteorites there are only about 22 chemical elements, the same monotonous catalogue, more or less complete, coming down at every meteoric fall. In our Earth, after a longer condensation and a more powerful chemical action, the catalogue became extended to 63 . In the Sun, after a still longer and more powerful action, the number of simple elements, judging from the fixed lines, must be several hundreds or several thousands. All the stars have different sets of fixed lines, showing that the modifications of matter which we call chemical elements are infinite in number. The same is seen in the planets. Mercury is nine times more dense than Saturn; and therefore the two are composed mainly of different elements. Greater heat might indeed expand the elements of Saturn more than those in Mercury, but not so much as nine times. This is impossible. All solids and liquids fly off into vapour long before even a double expansion. Moreover there is no estimating the millions of years which Saturn had to cool before the origin of Mercury. 
In that same Part I collected many facts to show the heterogeneity of matter, and how the peculiar elements of the Sun may possess many thousand times more chemical force than those in our Earth, and thus continue through periods illimitable to burn and to impart solar radiations. 'The same is true of the facts "dissociation," which have been insisted on so much. The oxide of gold is dissociated with truly an insignificant amount of heat; the oxide of mercury requires only a little more; hut the dioxide of carbon requires many tens of thousand times more heat for its dissociation, if indeed such dissociation by heat be at all possible. The peculiar elements of the sun may require we know not how much more. To say that the great heat of the sun must necessarily dissociate its elements, is to say what we do not know, and that in the face of the strongest reasons to the contrary.

I said there are three primitive forces whose origin is unknown -repulsion, chemical force, and gravity. But we perceive that from the operation of these three all the other special forces become manifest, through the great principle of the conversion of force. From the chemical force in ordinary burning come light and heat; from the same force in the galvanic battery come electricity and magnetism. The great force of cohesion is no exception. In the nebulous condition of the sun, at its greatest expansion, we cannot conceive of the operation of the cohesive force ; it was the product of the chemical force while overcoming the repulsire force of the nebula. This we see beautifully illustrated in oxygen and hydrogen. By the chemical force they are condeused into water; and Dr. Henry, of the Smithsonian Institution at Washington, first proved that the cohesion between the molecules of water is probably equal to that between the molecules of ice. In raising up a disk 1 inch square from the surface of water, a force equal to the weight of only 53 grains is required; but this is because loy after layer of the water is broken successively, like a strong cord when its many strands are broken one by one (his experiments are described in the fourth volume of the American Philosophical Society, Philadelphia),-whence he comes to the conclusion that the tensile force necessary to break a cubic inch of water, instead of being 53 grains, must be several hundred pounds. The difference between water and ice is not in the amonnt of cohesion, but in the fixed polarity of the molecules of ice, which by the action of heat are allowed in the water to turn in every direction.

$I$ have now pointed out in a very brief manner some of the many consequences flowing from the primitive diffusion of all matter through all space, and its slow condensation into stars 
by the chemical force. A few words more are necessary on the source of solar heat. My paper opposing the mechanical theories of solar heat, the fall of meteorites in the sun, and the fall of the materials of the sun towards its centre, was published in the 'Proceedings,' for 1867, of the Academy of Natural Sciences of Philadelphia ; and at its close I expressed a hope that the subject was put at rest for ever. But Mr. Croll has lately bronght out an addition to the mechanical theory. He admits the failure of the meteoric theory; and he concedes that the falling together by gravity of the materials of the sun would produce heat enough for only twenty millions of years-an insufficient period. But he supposes that two unluminous stars, each half the mass of our sun, must have been propelled together in opposite directions with velocities of 476 miles per second, and that their collision must have produced heat enough to supply the solar radiations for 50 millions of years. This vast amount of heat would have expanded our sun very widely in a nebulous condition; and by the falling together of this nebulous mass, heat enough wonld be produced to last 20 millions of years more of solar radiations. These 50 millions of years of heat derived from the collision, and these 20 millions derived from the falling together of the solar materials, make together 70 millions of years of solar light and heat. Many objections oppose all this.

1. If the solar heat for 20 millions of years would be produced by the falling together of the materials of the sun, then precisely the same amount of energy, or heat, would be consumed to expand the sun to its nebulous condition. And this would be subtracted from the heat of collision, 50 millions of years. Instead therefore of 70 millions of years, his period wonld remain only the original 50 millions from collision.

2. Mr. Croll has not proved, nor attempted to prove, that the heat of fifty millions of years would be sufficient to expand our sun far beyond the furthest planet, so that in condensing the solar system might be formed. When expanded only to the orbit of Neptune the sun was 14,000,000 less dense than hydrogen. By the Table of Marsh this would absorb and render latent a wonderful amount of latent heat, which $\mathrm{Mr}$. Croll must prove could be produced by his theoretical collision.

3. If our Sun were expanded beyond the orbit of Neptune by a sudden production of heat from any cause, then, in order to contract to nearly his present size, that heat must first radiate away. According to this scheme, the very fact of solar condensation presupposes the loss of heat by radiation. But, after the loss of all this heat which caused the expansion (the heat of 50 millions of years), none at all can be left for solar 
radiation after the formation of the planets. The theory leaves the solar system from its first creation in total darkness and inconceivably cold.

4. Mayer, the author of the meteoric theory, could not account for the former fused condition of our globe, and its present interior heat, by the fall of meteorites. $\mathrm{He}$ therefore supposed that two opaque stars had collided to form our Earth in a molten condition. Then he is bound to do the same with the other planets. In each case two planets must have collided, leaving the resulting planet in a nebulous state to form its satellites by condensation. And, mirabile dictu, all the collisions must have been so nicely adjusted that the resulting planets should rotate in the same direction on their axes, and revolve in the same plane and in the same direction round the sun, the same direction that the sun rotates, and nearly in the solar equatorial plane. The chances against all this scheme of collisions are infinite; and therefore it is impossible. Mr. Croll's theory is about the same. The sidereal systems are as orderly as our solar system. Our own sidereal system is composed chiefly of the ring of the galaxy, like the ring of the asteroids, and like the rings of Saturn. The ring in Lyra, with otber sidereal rings, and other regular forms of sidereal systems, shows the legitimate working of the nebular theory, but not the chance collisions of wandering stars.

5. Mr. Croll has to assume velocities for his colliding stars such as could not have been produced by the force of gravity. This is his own admission. But I have proved that gravity is the force which in the beginning put all the heavens and the earth in motion. No other force can be conceived to cause the stellar velocities ; and that force is sufficient*.

6. Mr. Croll's theory is, that originally alk the stars were dark and cold, and in the most rapid motion-far beyond the velocities which conld be produced by the force of gravity. The collisions, he says, have stopped them and made them shine: "The fixed stars are suns, and they are visible because they have lost their motions." But the fixed stars have not lost their motions. Every astronomer knows that they have their "proper motions," velocities more rapid than those of the planets. $61 \mathrm{Cygni}$ moves nearly 2000, and Arcturus nearly 3000 miles per minute.

7. There are sound reasons for believing that collisions among the stars is an impossibility. I have proved that the

* See 'The Origin of the Stars,' Tribner and Co., 1st London (from the 4th American) edition, and papers therein referred to; also the article on the Physical and Mathematical Principles of the Nebular Theory, in this Journal for April 1877. 
fixed stars in our sidereal system are all under each other's influence through gravity. Two stars approaching each other through gravity would at the same time be under the influence of other neighbouring stars, drawing them from the right line towards each other's centre. Therefore they would not collide; but they might approach very near to each other, so as to remain permanently within their powerfully gravitating force, and form a binary system. This is a mode of accounting for the ten thousand double and multiple stars already known, besides the mode of explanation by the nebular theory. Moreover the nebular theory necessarily gives very high velocities to all the stars, but to all in the same direction. If afterwards, by perturbation, they receive contrary directions, still the motions of any two precisely toward one another would be the most improbable of events.

Therefore the mechanical theory of stellar light and heat utterly fails in all its phases. The only true theory for the "ignes æterni" is that of chemical action. This also has been abruptly denied, but only by those entirely unacquainted with the foundations of that theory as explained by myself in 'The Origin of the Stars' and in subsequent papers.

XXIX. On Acoustic Repulsion. By V. Dvo̊̊ák*. With a Note by Prof. A. M. Mayer.

1. ACOUSTIC Repulsion of Resonators which are open at one end only.-In a previous article, "On Acoustic Attraction and Repulsion," I have conclusively proved by theoretic considerations, as well as by experiments, that the average pressure at the node in a column of air vibrating in stationary waves cannot be equal to zero as long as the amplitude of vibration is not infinitely small.

In a resonator open at one end, as, for example, a cylinder, we find a node at the closed end. In the interior of the cylinder near its closed end there exists a greater pressure than on the outer surface of this end which is touched by the outside air, as can easily be shown by means of a sensitive manometer.

To obtain resonance the opening of the cylinder is turned toward the source of the sound ; and the cylinder is then repelled by the excess of pressure within. Resonators not having a cylindrical form, but open at one end, are also subject to such repulsion. In my previous communication I have indicated

* From the American Journal of Science and Arts for July 1878. Translated from the Annalen der Physik und Chenie, Band iii. No. 3; dated Agram, November 19, 1877.

Phil. Mag. S. 5. Vol. 6. No. 36. Sept. 1878. 